\title{
Interaction and Size Effects in Open Nano-Electromechanical Systems
}

\section{B. Tanatar,* Valeriu Moldoveanu,* Radu Dragomir, and Stefan Stanciu}

The time-dependent transport of a 2D quantum wire (QW) connected to source/drain leads and electrostatically coupled to a singly-clamped InAs cantilever is investigated. The latter is placed above the nanowire and acts as a nanoresonator (NR) in the quantum regime. The vibron dynamics and the transport properties of this nano-electromechanical system (NEMS) are described within a generalized master equation approach which is exact with respect to the electron-vibron coupling. A detailed description of the electron-vibron coupling by taking into account its dependence on the wavefunctions of the quantum nanowire is introduced. It is shown that the tunneling processes in the QW trigger periodic oscillations of the average vibron number even in the absence of a bias. The time-dependent filling of the vibronic states changes as the nanoresonator is swept along the quantum wire.

\section{Introduction}

The observation of quantized vibrational modes of a nanomechanical resonator $(\mathrm{NR})^{[1,2]}$ and their controlled entanglement with the transport processes of nearby mesoscopic system (e.g carbon nanotubes (CNTs), quantum dots (QDs) ${ }^{[3]}$ ) stimulates a lot of theoretical work on time-dependent transport and sensing properties of nano-electromechanical systems. The electron-vibron electrostatic coupling is essentially controlled by changing the nanoresonator mass $M$ or the mode frequency $\omega_{0}$ through the oscillator length $\sqrt{\hbar / 2 M \omega_{0}}$ but also depends on the charge density of the mesoscopic system which reflects in turn the localization properties of its wavefunctions. Then each state of the mesoscopic system has its own coupling strength to the vibrational mode. These features cannot be captured by single-

\section{Prof. B. Tanatar}

Department of Physics, Bilkent University

Bilkent, 06800 Ankara, Turkey

E-mail: tanatar@fen.bilkent.edu.tr

Prof. V. Moldoveanu, Dr. R. Dragomir, S. Stanciu

National Institute of Materials Physics

PO Box MG-7, 077125 Bucharest-Magurele, Romania

E-mail:valim@infim.ro

S. Stanciu

Faculty of Physics, University of Bucharest

077125 Bucharest, Romania

The ORCID identification number(s) for the author(s) of this article can be found under https://doi.org/10.1002/pssb.201800443.

DOI: 10.1002/pssb.201800443 site single-level models which rely on a simple constant to parametrize the electron-vibron coupling.

In this work we highlight the effects of the position-dependent electron-vibron coupling and of the tunneling processes on the dynamics of the nanoresonator by theoretically modeling the transient vibron-assisted transport in a typical nanoelectromechanical setup. Our system consists of a singly clamped nanoresonator which is aligned to the $y$-axis such that its endpoint can vibrate above a twodimensional quantum wire of length $L_{\mathrm{x}}$ and width $L_{\mathrm{y}}$ (the setup is schematically presented in Figure 1). The nanowire is connected to the left and right leads having chemical potentials $\mu_{\mathrm{L}}$ and $\mu_{\mathrm{R}}$. In the absence of the electrostatic coupling to the QW the NR rests at the equilibrium distance $z_{0}$. Note that there is no tunneling between the two subsystems. However, when electrons tunnel through the nanowire the cantilever is driven out of equilibrium. One can also sweep the nanoresonator along the $x$-axis and then record the changes in its dynamics or in the transport properties of the QW. The geometry of the nanowire and the Coulomb interaction effects are also taken into account.

The theoretical studies on nano-electromechanical systems both in classical or quantum regimes rely on master equation approaches $^{[4-6]}$ or non-equilibrium Green's functions techniques. ${ }^{[7]}$ Starting from a single-level Anderson-Holstein (SLAH) Hamiltonian where the electron-vibron electrostatic coupling is parameterized by a simple constant $\lambda$, one performs the polaronic (Lang-Firsov) unitary transformation which diagonalizes the Hamiltonian and leads to vibronic sidebands.

Notably, the polaron transformation adds an operator-valued exponential to the lead-nanowire tunneling Hamiltonian which therefore becomes a more complicated object.

A different and much less explored route is to solve the master equation in the basis of the fully coupled system. To our best knowledge this route was first taken by Hubener and Brandes ${ }^{[8]}$ for the simple case of a single-level quantum dot coupled to a classical oscillator. They ended up with a Franck-Condon master equation which naturally embodies the overlap between different vibrational components of the interacting wavefunctions. The role of these position-dependent Franck-Condon terms on the mass sensing and transport properties of suspended CNTs has been later pointed out in the theoretical calculations of Remaggi et al. ${ }^{[9]}$ and Donarini et al. ${ }^{[10]}$ We stress that the suspended CNTs are used both as a conducting system 


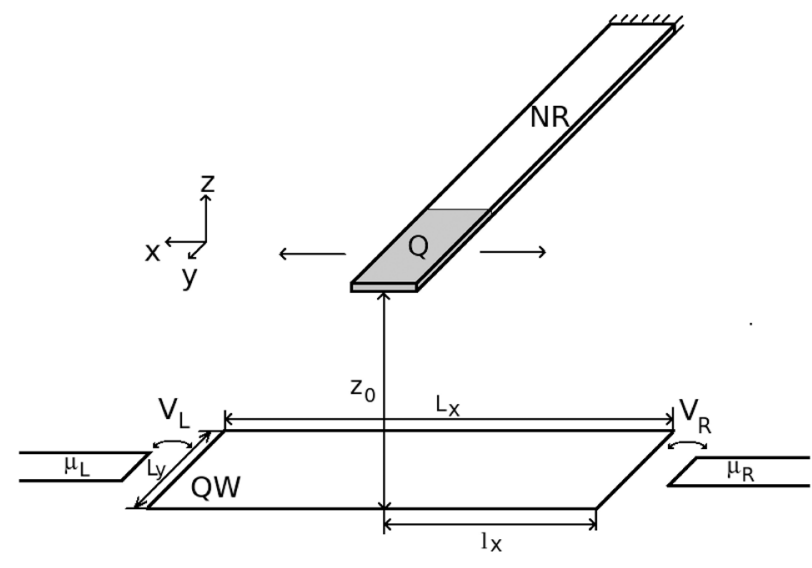

Figure 1. Sketch of the NEMS setup: the quantum wire lies in in the $x y$ plane and is connected to the left and right leads having chemical potentials $\mu_{\mathrm{L}}$ and $\mu_{\mathrm{R}}$. The free end of the NR accomodates the charge $Q$ and is placed above the QW. The NR can be shifted to the left or to the right on the $x$ axis, $I_{x}$ being the distance from the right edge of the $\mathrm{QW}$ to the NR. $V_{L, R}$ denote tunneling coefficients to the leads.

and as a nanoresonator driven by a microwave signal. Here the setup is very different as the quantum wire and the nanoresonator are separate systems.

The paper is organized as follows. In Section 2 we outline the transport formalism, Section 3 contains the numerical results and their analysis whereas Section 4 is left to conclusions.

\section{Formalism}

Here we introduce the main steps of the formalism. The Hamiltonian $H_{\mathrm{Qw}}$ of the quantum wire embodies the geometrical details and the effect of the Coulomb interaction. The single-particle eigenenergies and eigenfunctions are denoted by $\varepsilon_{i}$ and $\psi_{i}$, respectively. Then we introduce the Coulomb interaction and calculate numerically the low-energy interacting many-body configurations $|v\rangle$ and the associated eigenvalues $E_{v}$, such that $H_{\mathrm{QW}}|v\rangle=E_{v}|v\rangle$. The QW-NR system is described by the Hamiltonian

$H_{\mathrm{S}}=H_{\mathrm{QW}}+H_{\mathrm{NR}}+V_{\mathrm{el}-\mathrm{vb}}:=H_{\mathrm{S}}^{(0)}+V_{\mathrm{el}-\mathrm{vb}}$

where $H_{\mathrm{NR}}=\hbar \omega_{0} a^{\dagger} a$ and $V_{\mathrm{el}-\mathrm{vb}}$ stands for the electron vibron coupling which can be written as

$V_{\mathrm{el}-\mathrm{vb}}=\sum_{i \in Q W} v_{i} c_{i}^{\dagger} c_{i}\left(a^{\dagger}+a\right)$

Here $a^{\dagger} / a$ are the raising/lowering operators associated to the vibrational mode and $c_{i}^{\dagger} / c_{i}$ are creation/annihilation operators associated to a single-particle state $\psi_{i}$. Note that $H_{\mathrm{S}}^{(0)}|v, N\rangle=\left(E_{v}+N \hbar \omega_{0}\right)|v, N\rangle$ where we introduced the "free" states of the system in the absence of the electron-vibron interaction $|v, N\rangle:=|v\rangle \otimes|N\rangle,|N\rangle$ being the $N$-vibron state of the nanoresonator (i.e., $a^{\dagger} a|N\rangle=N|N\rangle$ ).

In Equation (2) $v_{i}$ denotes the electron-vibron coupling strength associated to the single-particle state $\psi_{i}$ of the non-interacting QW. Its explicit form is obtained by expanding the QW-NR electrostatic interaction about the equilibrium position $z_{0}$ and by quantizing the displacement $u=z^{\prime}-z_{0}$. The 1 st order term associated to the $i$-th single-particle state reads:

$$
\begin{aligned}
V_{i} & =\frac{e Q}{4 \pi \varepsilon_{0} \varepsilon_{r}} \int_{\mathrm{NR}} d \mathbf{r}^{\prime} \int_{\mathrm{QW}} d r N_{i}(r) \hat{u} \frac{\partial}{\partial z^{\prime}}\left|\mathbf{r}-\mathbf{r}^{\prime}\right|^{-1} \mid z^{\prime}=z_{0} \\
& =v_{i}\left(a^{\dagger}+a\right)
\end{aligned}
$$

where $N_{i}(\mathbf{r})=\left|\psi_{i}(\mathbf{r})\right|^{2}$ is the electronic density at site $i$ and $Q=e N_{\text {tip }}$ is the charge localized on the endpoint of the NR. Note that $v_{i} \sim \sqrt{\frac{\hbar}{2 M \omega_{0}}}$ but it also depends on the geometry of the nanowire. For simplicity we omitted the zero-order term which only induces a global shift of the eigenstates of the nanoresonator. Let us stress that in the SLAH model the conducting system is described as a single site and the analogue of Equation (3) will only capture the dependence on the equilibrium distance $z_{0}$ and on the oscillator length. However, this picture misses the electronic distribution within an extended conducting system such as the quantum wire we consider here. In Section 3.2 we shall calculate the vibron population and the fully interacting states of the nanomechanical system as a function of the nanoresonator's location along the nanowire; there we shall investigate in more detail the crucial role of the position-dependent electron-vibron coupling.

Since the electron-vibron interaction conserves the electronic occupation of the quantum wire it follows that for any manybody (MB) configuration $v$ one gets a subspace of fully interacting states $\{|v, s\rangle\}$ which only differ by the weights $A_{\mathrm{sN}}^{(v)}$ of different vibron states $|N\rangle$ :

$|v, s\rangle=|v\rangle \otimes\left\{\sum_{N} A_{\mathrm{sN}}^{v}|N\rangle\right\}:=|v\rangle \otimes\left|s_{v}\right\rangle$

where the notation $s_{v}$ recalls that the overlap of different vibrational components $|N\rangle$ depends on the many-body configuration. The number of vibronic states $s$ must be limited to a cut-off value $N_{\text {eff }}$ such that $N=1,2, \ldots N_{\text {eff }}$. Note that for each many body configuration $v$ the coefficients $A_{\mathrm{sN}}^{(v)}$ define a unitary transformation between $\{|N\rangle\}$ and $\left\{\left|s_{v}\right\rangle\right\}$. In other words the Hamiltonian of the QW-NR systems is block diagonal w.r.t $|v\rangle$. The eigenvalues $\mathcal{E}_{\mathrm{vs}}$ of the coupled electron-vibron system are defined by $H_{\mathrm{S}}|v, s\rangle=\mathcal{E}_{\mathrm{vs}}|v, s\rangle$.

The transfer Hamiltonian describing the lead-QW coupling has a standard form:

$H_{\mathrm{T}}=\sum_{a} \sum_{k, \sigma} \sum_{i \in \mathrm{QW}}\left(V_{i, k \sigma}^{\alpha} c_{i}^{\dagger} c_{k a \sigma}+\right.$ h.c. $)$

where $(k, \sigma)$ stand for the momentum and spin of an electron in the reservoir $\alpha$ and $V_{i, k \sigma}^{\alpha}$ is the tunneling strength. We assume for simplicity that the tunneling processes conserve the spin such and does not depend on $k$ and we shall use the simplified notation $V_{\mathrm{L}}=V_{\mathrm{R}}=V$ for the tunneling coefficients.

The dynamics and the transport properties of the system are calculated from the master equation of the reduced density operator $\rho(t)=\operatorname{Tr}_{\text {leads }}\{W(t)\}$ where $W(t)$ is the density operator 
of the whole system which solves $i \hbar \dot{W}(t)=\left[H_{\mathrm{S}}+H_{\mathrm{T}}+H_{\text {leads }}, W\right]$. The leads are suddenly coupled at some initial time $t_{0}$ and $\rho\left(t_{0}\right)=\left|v_{0}, N=0\right\rangle\left\langle v_{0}, N=0\right|$ for some initial configuration $v_{0}$.

Within the Born-Markov approximation the master equation reads:

$\dot{\rho}(t)=-\frac{i}{\hbar}\left[H_{\mathrm{S}}, \rho(t)\right]-L_{\text {leads }}[\rho(t)]-L_{\kappa}[\rho(t)]$

where $L_{\text {leads }}$ takes into account the contribution of the particle reservoirs (i.e., the leads) and $L_{\kappa}$ describes the damping of vibrons due to a thermal reservoir.

By straightforward and standard calculations one finds that the Lindblad-like terms are expressed in a compact form

$L_{\text {leads }}[\rho(t)]=\frac{\pi}{\hbar}\left(\sum_{a, \sigma}\left[A_{\alpha \sigma}, B_{\alpha \sigma} \rho(t)-\rho(t) \mathcal{D}_{\alpha \sigma}^{\dagger}\right]+\right.$ h.c. $)$

In the basis of fully interacting states $|v, s\rangle$ the operators $A, B$ and $D$ are given as follows:

$A_{\alpha \sigma}=\sum_{v s, v^{\prime} s^{\prime}} T_{v s, v^{\prime} s^{\prime}}^{\alpha \sigma}|v s\rangle\left\langle v^{\prime} s^{\prime}\right|$

$B_{a \sigma}=\sum_{\lambda r, \lambda^{\prime} r^{\prime}}\left(1-f_{\alpha}\left(\mathcal{E}_{\lambda^{\prime} r^{\prime}}-\mathcal{E}_{\lambda r}\right)\right) \bar{T}_{\lambda r^{\prime}, \lambda r}^{\alpha \sigma}|\lambda r\rangle\left\langle\lambda^{\prime} r^{\prime}\right|$

$\mathcal{D}_{\alpha \sigma}=\sum_{\lambda r, \lambda^{\prime} r^{\prime}} f_{a}\left(\mathcal{E}_{\lambda r}-\mathcal{E}_{\lambda^{\prime} r^{\prime}}\right) T_{\lambda r, \lambda^{\prime} r^{\prime}}^{a \sigma}|\lambda r\rangle\left\langle\lambda^{\prime} r^{\prime}\right|$

where we introduced the Fermi functions $f_{\alpha}(E)$ and jump operators between pairs of fully interacting states $\left(D_{a \sigma}\right.$ is the density of states of the lead $\alpha$ ):

$$
T_{v s, v^{\prime} s^{\prime}}^{a \sigma}=\sqrt{D_{a \sigma}} \sum_{i \in \mathrm{QW}} V_{i, \sigma}^{a}\left\langle v\left|c_{i}^{\dagger}\right| v^{\prime}\right\rangle \cdot\left\langle s_{v} \mid s_{v^{\prime}}^{\prime}\right\rangle
$$

The thermal damping term reads simply as

$L_{\kappa}=\frac{\kappa}{2}\left(a^{\dagger} a \rho+\rho a^{\dagger} a-2 a \rho a^{\dagger}\right)$

The above master equation describes only the sequential tunneling events, i.e., processes up to the second order in the coupling to the leads $V_{L, R}$. This limits the GME approach to weak coupling to the leads but it does not impose any conditions on the frequency of the nanoresonator. Howewer, as long as the leads are weakly coupled and the bias window contains some states of the nanowire the main contribution to the transport comes from the sequential tunneling and there is no need to include higher order terms in the dissipator $L_{\text {leads }}$. Let us note in particular that we solve our master equation without assuming the rotating wave approximation (RWA).

The master equation will be solved with respect to the fully interacting states $|v, s\rangle$ of the QW-NR system. Here "fully interacting" means that both the Coulomb interaction within the
QW and the electron-vibron coupling are taken into account. The main point here is that along the derivation of the master equation the argument of the Fermi functions is given by the energy difference between two fully interacting states (e.g., $\left.f_{a}\left(\mathcal{E}_{v s}-\mathcal{E}_{v^{\prime} s^{\prime}}\right)\right)$. Essentially this means that the electron-vibron coupling renormalizes the tunneling energy $E_{v}-E_{v^{\prime}}$ between two many-body configurations of the mesoscopic system. Note that the numbers of electrons for the pair $v, v^{\prime}$ obey the identity $\mid n(v)-n\left(\left(v^{\prime}\right) \mid=1\right.$ (tunneling or tunneling out events) and that the scalar product between two vibrational components $s_{v}$ and $s_{v^{\prime}}$ is nothing but the Franck-Condon factor:

$$
\left\langle s^{\prime}{ }_{v^{\prime}} \mid s_{v}\right\rangle=\sum_{N} \overline{A_{s^{\prime} N}^{\left(v^{\prime}\right)}} A_{s N}^{(v)}
$$

As a particular case one can choose $\mu_{\mathrm{L}}$ and $\mu_{\mathrm{R}}$ such that $f_{\mathrm{L}}(E)=1-f_{\mathrm{R}}(E)=1$ for all energies considered in the numerical calculations. Then it is easy to see that that $\mathcal{B}_{c \sigma}=$ $\mathcal{A}_{c \sigma}^{\dagger}$ and that $\mathcal{D}_{v \sigma}=\mathcal{A}_{v \sigma}$. Consequently, the leads' dissipative term acquires the well known Lindblad form

$\mathcal{L}_{a, \sigma}[\rho]=\mathcal{A}_{a, \sigma} \mathcal{A}_{a, \sigma}^{\dagger} \rho+\rho \mathcal{A}_{a, \sigma} \mathcal{A}_{a, \sigma}^{\dagger}-2 \mathcal{A}_{a, \sigma}^{\dagger} \rho \mathcal{A}_{a, \sigma}$

Now let us compute the observables in terms of the matrix elements of $\rho(t)$. We denote by $Q_{\mathrm{S}}=e N_{\mathrm{S}}=e \sum_{i} c_{i}^{\dagger} c_{i}$ the charge operator in the sample. The two time-dependent currents are identified and calculated from the continuity equation:

$\frac{d}{d t} Q_{\mathrm{S}}(t)=e \operatorname{Tr}\left\{N_{\mathrm{S}} \frac{d}{d t} \rho(t)\right\}=J_{\mathrm{L}}(t)-J_{\mathrm{R}}(t)$

where $\operatorname{Tr}$ denotes the average with respect to the fully interacting basis $|s, N\rangle$. By convention the current in the left contact $J_{L}$ is positive if electrons flow from the contact into the $\mathrm{QD}$, while the current in the right contact $J_{R}$ is positive if electron leave the energy levels of the QD. Each current can be identified by noticing that $\mathcal{L}_{\text {leads }}=\mathcal{L}_{\mathrm{L}}+\mathcal{L}_{\mathrm{R}}$.

The vibron number is calculated as $N_{v}=\operatorname{Tr}\left\{\rho(t) a^{\dagger} a\right\}$, while the electron number $N_{\mathrm{S}}=\operatorname{Tr}\left\{N_{\mathrm{S}} \rho(t)\right\}$. Finally the displacement of the nanoresonator $d=\sqrt{\frac{\hbar}{2 M \omega_{0}}} \operatorname{Tr}\left\{\left(a^{\dagger}+a\right) \rho(t)\right\}$.

As for the matrix elements of the reduced density operator we used the inverse transformation $|v, N\rangle=\sum_{s} \overline{A_{s N}}|s, N\rangle$ to switch back to the "free" basis which is more convenient for discussions. In particular the population of $\mathrm{N}$-vibron states containing is defined as:

$P_{\mathrm{N}}=\sum_{v}\langle v, N|\rho(t)| v, N\rangle$

\section{Results}

The two-dimensional quantum wire is described by a lattice Hamiltonian. The corresponding hopping energy $t_{\mathrm{S}}=\hbar^{2} / 2 m^{*} \tau$ is related to the effective electron mass $m^{*}$ and to the lattice constant $\tau$. More specifically we considered an InAs quantum wire of length $L_{x}=75 \mathrm{~nm}$ and width $L_{Y}=10 \mathrm{~nm}$. Unless 
otherwise stated the temperature of the system is $T=50 \mathrm{mK}$; with the present cooling techniques such a regime was already achieved in experiments.

Our calculations were performed in the so-called slow vibration regime, ${ }^{[6]}$ which corresponds to a large ratio between the tunneling rate $\Gamma=2 \pi V^{2} D$ to the leads and the frequency of the nanoresonator $\gamma=\Gamma / \hbar \omega_{0}$. D is the density of states in the leads and $V=V_{\mathrm{L}}=V_{\mathrm{R}}$ is the tunneling coefficient between the leads and the nanowire. The bending mode of an InAs cantilever is of the order of hundreds of $\mathrm{MHz}$ which brings $\hbar \omega_{0}$ down to few $\mu \mathrm{eVs}$. For our numerical calculations $\gamma \approx 20$.

The geometry of the nearby nanoresonator cannot be easily simulated but a minimal model still allows us to calculate the electrostatic coupling to the QW in some detail. We consider a one-dimensional cantilever oriented along the $y$-axis. A collection of few sites located above the $\mathrm{QW}$ at an equilibrium distance $z_{0}$ (on the z-axis) simulate the endpoint of the cantilever. The semi-infinite leads attached to the NW are also described by discrete Laplacians. A two-dimensional lead is just a bunch of 1D "channels" coupled at consecutive sites of the sample edge. In the numerical calculations we considered four-channel leads. For simplicity we selected the chemical potentials of the leads such that no more than two electrons participate in transport. Then spin-up and spin-down electrons coming from the leads will then occupy the lowest single-particle state which is mostly localized in the center of the QW but also extends towards its endpoints. The master equation is solved numerically by using a 4-point Runge-Kutta method. We find that both the diagonalization procedure and the transport simulations are stable if 10 vibron states are included in the numerical calculations.

\subsection{Vibron Dynamics in an Unbiased System}

We find that the electron-vibron coupling drives the nanoresonator out-of-equilibrium even in the absence of an applied bias. In this configuration the leads are coupled to the system at instant $t_{0}=0$ but their chemical potentials are equal such that $\mu_{\mathrm{L}}=\mu_{\mathrm{R}}=\mu_{0}$ and electrons tunnel to the system until the system is fully charged and a steady-state state is reached.

Figure 2a shows the evolution of the average vibron number for several values of the equilibrium distance $z_{0}$ between the quantum wire and the resonator. The latter is centered with respect to the $x$-axis such that $l_{x}=37.5 \mathrm{~nm}$, where $l_{x}$ denotes the position of the NR on the $x$-axis. We selected $\mu_{\mathrm{L}}$ and $\mu_{\mathrm{R}}$ such that the wire accumulates at most one $\left(\mu_{0}=35 \mathrm{meV}\right)$ or two electrons $\left(\mu_{0}=80 \mathrm{meV}\right)$. This charging follows from the fact that the spin-degenerate lowest singleelectron energies are $E_{\uparrow}=E_{\downarrow}=31.85 \mathrm{meV}$, while the energy of the doubly occupied state is $E_{\uparrow \downarrow}=77.25 \mathrm{meV}$. The vibron number displays periodic oscillations for all configurations, in spite of the fact that the charge on the $Q W$ settles down rapidly (not shown) to $Q=1$ or $Q=2$. These oscillations are due to the electron-vibron interaction $\left\langle v, N\left|V_{\mathrm{el}-\mathrm{vb}}\right| v, N^{\prime}\right\rangle$ which couples "neighbor" vibron states (i.e., $\left.N-N^{\prime}= \pm 1\right)$ and therefore generates coherences in the master equation. In the long time limit the oscillations of $N_{v}$ are damped by the coupling to the thermal bath. However, the vibron number reaches a non-vanishing steady-state value. The damping rate $\kappa=0.1 V_{0}$, where $V_{0}$ is the interaction strength associated to the pair of states $|1, N=1\rangle$ and $|1, N=0\rangle$.
In the case of double-occupancy $Q=2$ we show results for two values of the equilibrium distance $z_{0}$. As expected, when the NR approaches the system by just $10 \mathrm{~nm}$ (from $z_{0}=160 \mathrm{~nm}$ to $z_{0}=150 \mathrm{~nm}$ ) the vibron number increases as the electrostatic coupling is enhanced. For single-occupancy configuration $(Q=1)$ the electron-vibron coupling is reduced and one has to set the initial position of the nanoresonator to $z_{0}=125 \mathrm{~nm}$ in order to obtain oscillations with an amplitude around 0.5.

Further information could be extracted by looking at the populations of $N$-vibron states. Figure $2 \mathrm{~b}$ captures the out-ofphase oscillations of $P_{0}$ on one hand and $P_{1,2,3}$ on the other hand. We also see that the main contribution to the vibronic populations is due to the one and two vibron states while $P_{3}$ can be neglected. The transient filling of the vibronic states reflects the "climbing" of the harmonic oscillator states due to the electron-vibron coupling. Indeed, $P_{0}$ reaches the 1 st maxima earlier than $P_{1}$ which in turn increases must faster than $P_{2}$. Moreover, each population has its own oscillation period. This is somehow expected because the electron-vibron couplings between neighbor states depend on the vibron numbers.

The calculations discussed above were obtained starting from the initial state $|0, N=0\rangle$ (that is there are no electrons or vibrons in the system before the coupling to the leads is switched on). Clearly, the steady-state quantities do not depend on the choice of the initial state.

\subsection{The Dependence of the Vibron Number on the Location of the NR on the $x$-Direction}

The numerical results were obtained in the transport regime, that is the system is subjected to a bias such that the lowestenergy states with at most one electron are available for tunneling processes. Similar results were obtained for a bias which activates as well the two-electron state $E_{\uparrow \downarrow}$. If the NR is placed above the center of the nanowire (i.e., for $l_{x, \text { tip }}=37.5 \mathrm{~nm}$ ) it will interact strongly with the charge localized on lowest energy single-particle state which is mostly localized there. When the nanoresonator moves along the $x$-axis towards the endpoints of the wire the electrostatic potential decreases.

To check if this simple fact has consequences on the dynamics of the system we present in Figure $3 \mathrm{a}$ and $\mathrm{b}$ the populations $P_{N}$ of the $N$-vibron states as a function of time for two locations of the nanoresonator on the $x$-axis. The populations $P_{2,3,4,5}$ of excited vibron states presented in Figure $3 \mathrm{~b}$ settle down to smaller values than the ones shown in Figure $3 \mathrm{a}$ while $P_{0,1}$ slightly increase. A similar effect is noticed on the average vibron number (see Figure 4) which drops from $N_{v}=1.4$ to $N_{v}=1.25$. The population $P_{1}$ depends very weakly on $l_{x}$ and was therefore omitted.

Note that the populations and the vibron number still display small oscillations but their amplitude is greatly reduced when compared to the unbiased configuration.

We also find that the displacement of the NR oscillates for a long time (up to $2 \mu \mathrm{s}$ ) and settles down to a different value when $l_{x}$ is varied (not shown). In contrast, if the chemical potentials of the leads are equal the NR always returns to its equilibrium value $z_{0}$. Let us emphasize that for the parameters selected here the displacement of the NR is of orders of femtometers so it can be detected in experiments. 

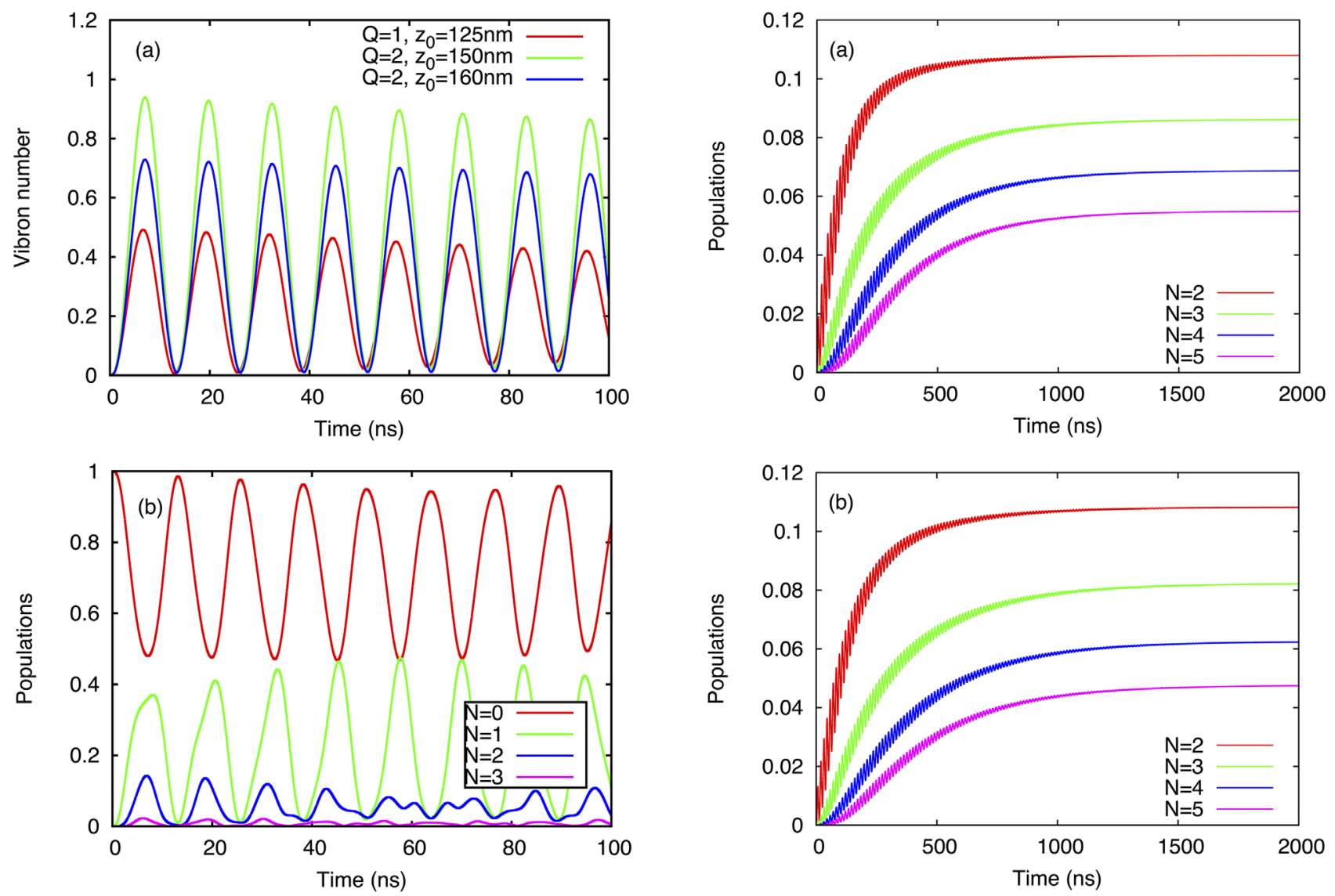

Figure 2. a) The average vibron number for several values of the equilibrium distance $z_{0}$ to an unbiased quantum wire. Both single $(Q=1)$ and double $(Q=2)$ occupancies are considered; the selected values for the chemical potentials $\mu_{\mathrm{L}}, \mu_{\mathrm{R}}$ are indicated in the text. b) The populations of $N$-vibron states for the double-occupacy configuration at $z_{0}=150 \mathrm{~nm}$. Other parameters: $\omega_{0}=500 \mathrm{MHz}, M=2.5 \times 10^{-15} \mathrm{~kg}$.

Finally, we investigate the weights $\left|A_{\mathrm{sN}}^{(v)}\right|^{2}$ of various vibron states $|N\rangle$ in a fully interacting state for different locations of the NR both w.r.t. the $x$ and $z$ directions. The fully interacting eigenstates $|v, s\rangle$ and their corresponding eigenvalues $\varepsilon_{v s}$ are calculated by numerical diagonalization. The stability of the diagonalization procedure is reached if by adding more vibron states in the calculation the weights remain unchanged. We can easily guess the connection between $\left|A_{\mathrm{sN}}^{(v)}\right|{ }^{2}$ and the mixing of different non-interacting states $|v, N\rangle$ in a fully interacting state. On one hand, if the relevant electron-vibron interaction matrix elements are much smaller than the gap between two consecutive states $\{|v, N\rangle,|v, N \pm 1\rangle\}$ which always equals $\hbar \omega_{0}$ one expects that for any $\left|S_{\nu}\right\rangle$ there exists a vibron state $|\tilde{N}\rangle$ such that its weight is much larger than any other weights. This situation then corresponds to a weak mixing due to the electronvibron interaction, as the off-diagonal perturbation is too small to induce a strong mixing. We find that this is the case for the lowest single-electron spin-up state $|\sigma=\uparrow, s=1\rangle$ in which the weight of $|\uparrow, N=0\rangle$ is around 0.95 for both $z_{0}=150 \mathrm{~nm}$ and $z_{0}=125 \mathrm{~nm}$. On the other hand for the excited vibronic states (i.e., for $s>1$ ) the relevant weights are spread over many vibron

Figure 3. The population of $N$-vibron states for two locations of the NR on the $x$-axis. a) $I_{x}=37.5 \mathrm{~nm}$ - centered, b) $I_{x}=2 \mathrm{~nm}$ - right. Other parameters: $\omega_{0}=500 \mathrm{MHz}, M=2.5 \times 10^{-15} \mathrm{~kg}, z_{0}=150 \mathrm{~nm}, \mu_{\mathrm{L}}=35$ $\mathrm{meV}, \mu_{\mathrm{L}}=25 \mathrm{meV}$.

numbers $N$. This can be seen in Figure 5 which presents the weights of the vibron states $|N\rangle$ (for $N=0, \ldots, 9$ ) corresponding to the 2nd (see Figure 5a and c) and the 4th (Figure $5 \mathrm{~b}$ and d) fully interacting states. Each figure contains the weights for three

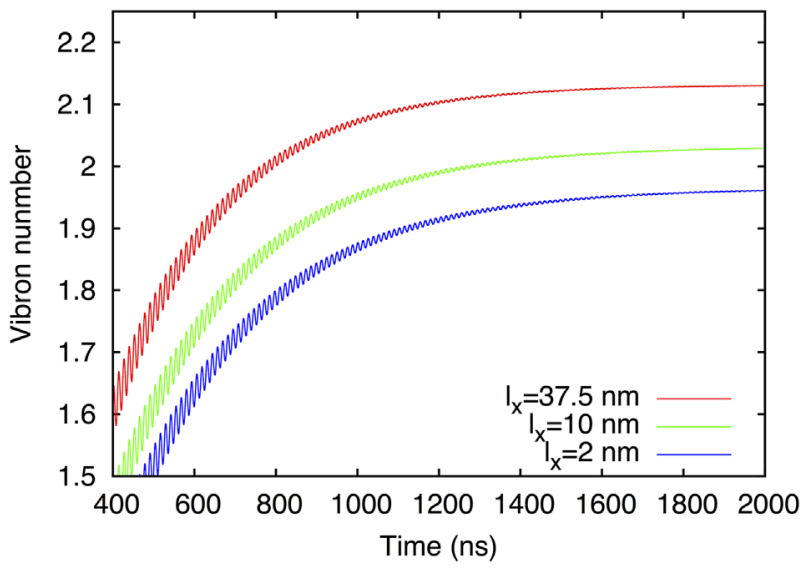

Figure 4. Vibron number for different NR positions along the $x$-axis. Initial state: $|n=0, N=1\rangle, \omega_{0}=500 \mathrm{MHz}, M=2.5 \times 10^{-15} \mathrm{~kg}, z_{0}=150 \mathrm{~nm}$, $\mu_{\mathrm{L}}=35 \mathrm{meV}, \mu_{\mathrm{R}}=25 \mathrm{meV}$. 


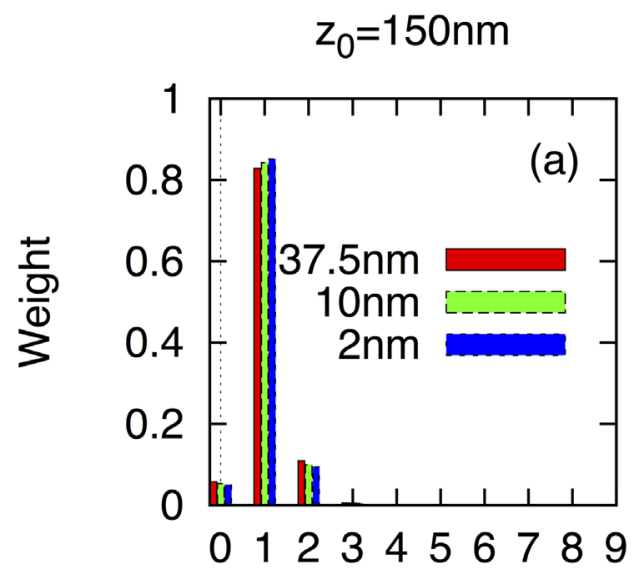

$\mathrm{z}_{0}=150 \mathrm{~nm}$
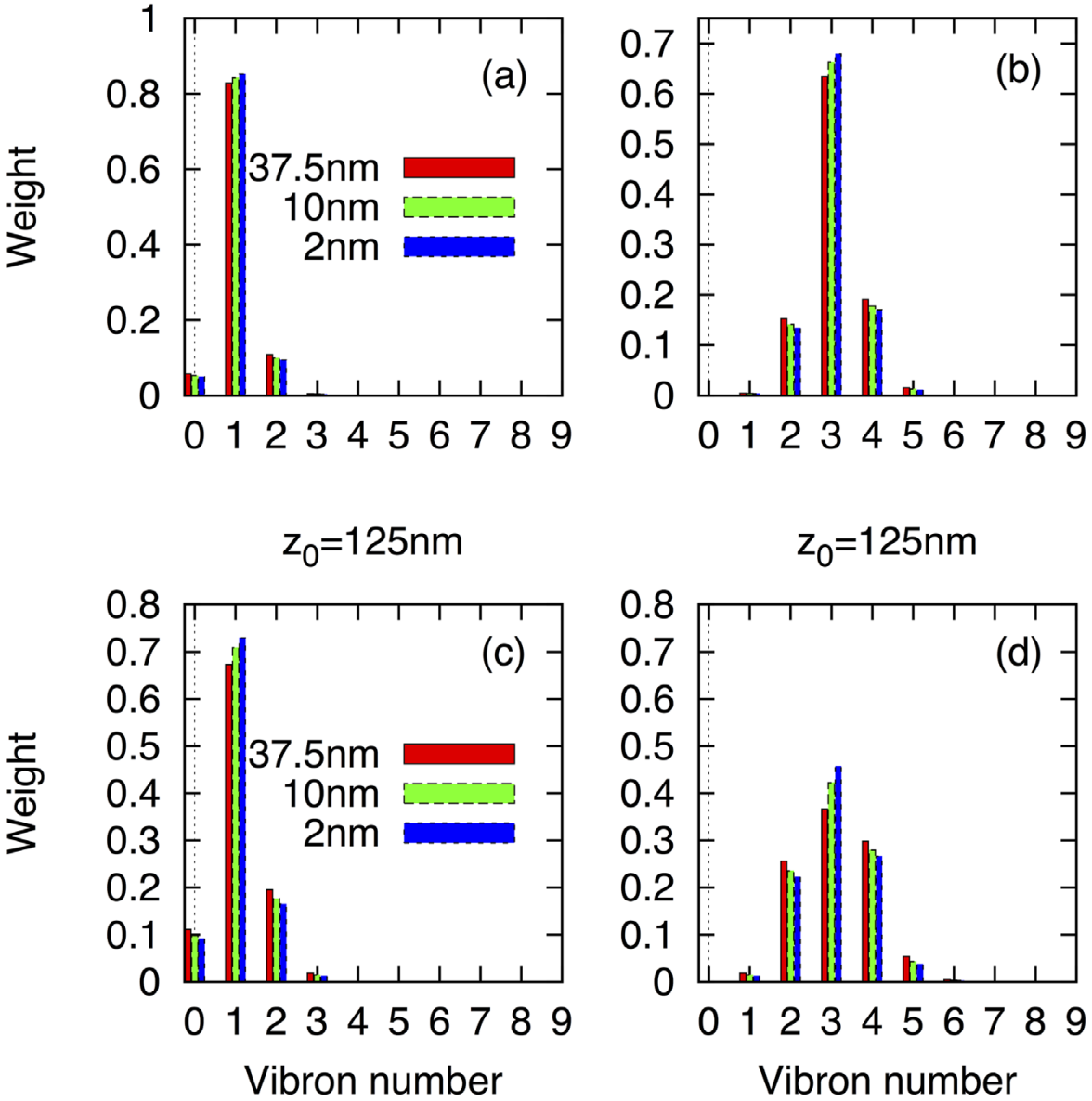

Figure 5. The weights $\left|A_{s N}^{(v)}\right|^{2}$ of the "free" states $|v, N\rangle$ in a fully interacting state $|v, s\rangle$ for different equilibrium positions $z_{0}$ and locations $I_{x}$ along the $x$ axis. Parts (a and c) - the state $|\uparrow, s=2\rangle$; (b) and (d) - the state $|\uparrow, s=4\rangle$. Other parameters: $\omega_{0}=500 \mathrm{MHz}, M=2.5 \times 10^{-15} \mathrm{~kg}, d_{0}=150 \mathrm{~nm}$, $\mu_{\mathrm{L}}=35 \mathrm{meV}, \mu_{\mathrm{R}}=25 \mathrm{meV}$.

positions of the NR along the $x$-axis. A non-vanishing mixing is noticed even for the second state in Figure 5 a although the onevibron state carries the dominant weight, while the 4th state collects contributions from higher vibron numbers, especially at $z_{0}=125 \mathrm{~nm}$. We also notice that as the NR approaches the right edge of the quantum wire (i.e., for $l_{x}=2 \mathrm{~nm}$ ) the mixing of the non-interacting states reduces. For higher energy states the mixing becomes even more pronounced as the electron-vibron coupling between pairs of non-interacting states scales like $\sqrt{N}$.

Now let us comment on the backaction effect of the NR on the transport properties. For the frequencies considered here energies $N \hbar \omega_{0}$ are very small (i.e., few $\mu \mathrm{eV}$ ) so the vibronic states included in the calculations are well within the bias window such that $f_{a}\left(\mathcal{E}_{v s}-\mathcal{E}_{v^{\prime} s^{\prime}}\right)$ is either 0 or 1 . Then the Lindblad Equation (13) holds. Moreover, we find that the current passing through the nanowire does not detect the electronvibron coupling. This is explained by noticing that even if the electron-vibron coupling brings in excited vibron states the associated tunneling processes still contribute to the current as long as the energy differences $\mathcal{E}_{v s}-\mathcal{E}_{v^{\prime} s^{\prime}}$ are within the bias window. In order to capture a change in the transport properties due to the electron-vibron coupling one has to increase the level spacing of the vibronic states, that is to increase the frequency $\omega_{0}$. This is the case in molecular transistor where $\hbar \omega_{0}$ reaches the $\mathrm{meV}$ range and the Franck-Condon blockade can be measured.

A brief discussion on the available frequency range for various NEMS is useful here. A transverse oscillation mode around $39 \mathrm{GHz}$ has been reported for a suspended $\mathrm{CNT}^{[11]}$ while stretching modes can go up to $200 \mathrm{GHz} .{ }^{[12]}$ Notably, short CNTs were shown to display extremely high frequency strain-tunable bending modes (few hundred GHz). ${ }^{[13]}$ Preliminary calculations show that if the NR frequency increases to tens or even hundreds of $\mathrm{GHz}$ the "ladder" of harmonic oscillator levels $\varepsilon_{v N}^{(0)}=E_{v}+N \hbar \omega_{0}$ can be scanned by varying the chemical potentials of the leads. Consequently, the current depends on the population of these vibronic states. This regime will be studied in a future work.

Let us stress that the results presented in Section 3.2 are not compatible with the notion of a single and position-independent electron-vibron coupling as the one assumed in the SLAH model. Indeed, the fact that the occupation of different vibron states and the average vibron number depend on the location of the NR on the $x$-axis cannot be captured unless the electron- 
vibron coupling depends explicitly on the electronic distribution of the wavefunctions of the quantum wire.

\section{Conclusions}

We presented a Franck-Condon master equation for nanoelectromechanical systems by treating the Coulomb interaction and the electron-vibron coupling on equal footing. The latter depends both on the position of the nanoresonator but also on the localization properties of the single-particle states in the quantum wire. The eigenfunctions of the nano-electromechanical system are found by using configuration-interaction methods. Our simulations were performed for a set of parameters which are encountered in typical transport measurements on quantum wires. The QW-NR setup illustrates both the dependence of the electron-vibron coupling on the spacial extend of the wavefunctions and the different sensing efficiency of the NR as a function of its location above the conducting mesoscopic system. Otherwise stated, we explicitly show that in general it is not appropriate to parametrize the electrostatic coupling by a simple/single constant.

We calculated the populations associated to different vibron numbers and investigated their dynamics for various locations of the NR on the $x$-axis. The coupling of the nanoresonator to a thermal bath limits the number of vibronic states excited by the current passing through the wire and drives the system to a steady state. If the states participating to the transport are well within the bias window the nanowire cannot detect the vibron dynamics. The role of the open quantum wire remains however crucial as it sets the NR into motion and changes its equilibrium position.

\section{Acknowledgements}

VM, RD, and SS acknowledge financial support by the CNCS-UEFISCDI Grant PN-III-P4-ID-PCE-2016-0084. BT and VM were also supported by TUBITAK Grant No. $117 \mathrm{~F} 125$.

\section{Conflict of Interest}

The authors declare no conflict of interest.

\section{Keywords}

nano-electromechanical systems, quantum transport

Received: August 27, 2018

Revised: January 4, 2019

Published online: February 14, 2019

[1] A. D. O'Connel, M. Hofheinz, M. Ansmann, R. C. Bialczak, M. Lenander, E. Lucero, M. Neeley, D. Sank, H. Wang, M. Weides, J. Wenner, J. M. Martinis, A. N. Cleland, Nature 2010, 463, 697.

[2] J. D. Teufel, T. Donner, D. Li, J. W. Harlow, M. S. Allman, K. Cicak, A. J. Sirois, J. D. Whittaker, K. W. Lehnert, R. W. Simmonds, Nature 2011, 475, 359

[3] M. Poot, H. S. J. van der Zant, Phys. Rep. 2012, 511, 273.

[4] D. W. Utami, H.-S. Goan, G. J. Milburn, Phys. Rev. B 2004, 70, 075303.

[5] D. A. Rodrigues, A. D. Armour, New J. Phys. 2005, 7, 251.

[6] G. Piovano, F. Cavaliere, E. Paladino, M. Sassetti, Phys. Rev. B 2011, $83,245311$.

[7] K. F. Albrecht, A. Martin-Rodero, R. C. Monreal, L. Mühlbacher, A. Levy Yeyati, Phys. Rev. B 2013, 87, 085127.

[8] H. Hubener, T. Brandes, Phys. Rev. B 2007, 80, 155437.

[9] F. Remaggi, N. T. Ziani, G. Dolcetto, F. Cavaliere, M. Sassetti, New J. Phys. 2013, 15, 083016.

[10] A. Donarini, A. Yar, M. Grifoni, New J. Phys. 2012, 14, 023045.

[11] E. A. Laird, F. Pei, W. Tang, G. A. Steele, L. P. Kouwenhoven, Nano Lett. 2012, 12, 193.

[12] P. Weber, H. L. Calvo, J. Bohle, K. Gos, C. Meyer, M. R. Wegewijs, C. Stampfer, Nano Lett. 2015, 15, 4417.

[13] J. O. Island, V. Tayari, A. C. McRae, A. R. Champagne, Nano Lett. 2012, 12, 4564 